\title{
Genome-wide identification of CBL family and expression analysis of $C B L s$ in response to potassium deficiency in cotton
}

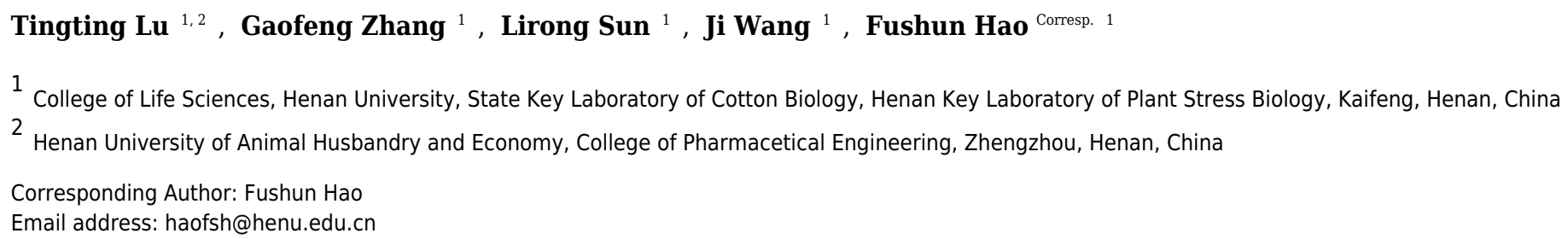

Calcineurin B-like $(\mathrm{CBL})$ proteins, as calcium sensors, play pivotal roles in plant responses to diverse abiotic stresses and in growth and development through interaction with CBLinteracting protein kinases (CIPKs). However, knowledge about functions and evolution of CBLs in Gossypium plants is scarce. Here, we conducted a genome-wide survey and identified 13, 13 and 22 CBL genes in the progenitor diploid Gossypium arboreum and Gossypium raimondii, and the cultivated allotetraploid Gossypium hirsutum, respectively. Analysis of physical properties, chromosomal locations, conserved domains and phylogeny indicated rather conserved nature of CBLs among the three Gossypium species. Moreover, these CBLs have closer genetic evolutionary relationship with the CBLS from cocoa than with those from other plants. Most CBL genes underwent evolution under purifying selection in the 3 Gossypium plants. Additionally, nearly all $G$. hirsutum CBL (GhCBL) genes were expressed in the root, stem, leaf, flower and fiber. Many GhCBLs were preferentially expressed in the flower while several GhCBLs were mainly expressed in roots. Expression patterns of GhCBL genes in response to potassium deficiency were also studied. The expression of most GhCBLs were moderately induced in roots after treatments with low-potassium stress. Yeast two-hybrid experiments indicated that GhCBL1-2, GhCBL1-3, GhCBL4-4, GhCBL8, GhCBL9 and GhCBL10-3 interacted with GhCIPK23, respectively. Our results provided a comprehensive view of the CBLs and valuable information for researchers to further investigate the roles and functional mechanisms of the CBLs in Gossypium. 
1 Title page

2 Title:

3 Genome-wide identification of CBL family and expression analysis of $C B L s$ in response to

4 potassium deficiency in cotton

5 Authors:

6 Tingting Lu ${ }^{1,2 *}$, Gaofeng Zhang ${ }^{*}$, Lirong Sun ${ }^{1}$, Ji Wang ${ }^{1}$ and Fu-Shun Hao ${ }^{1}$

$7 \quad{ }^{1}$ State Key Laboratory of Cotton Biology, Henan Key Laboratory of Plant Stress Biology,

8 College of Life Sciences, Henan University, Kaifeng 475004, China

$9 \quad{ }^{2}$ Henan University of Animal Husbandry and Economy, Zhengzhou 450011, China

$10 *$ These authors contributed equally to this work

11 Corresponding author

12 Fu-Shun Hao, haofsh@henu.edu.cn 
13 Genome-wide identification of CBL family and expression analysis of $C B L s$ in response to potassium deficiency in cotton

15 Tingting Lu ${ }^{1,2^{*}}$, Gaofeng Zhang ${ }^{1^{*}}$, Lirong Sun ${ }^{1}$, Ji Wang ${ }^{1}$ and Fu-Shun Hao ${ }^{1}$

1 State Key Laboratory of Cotton Biology, Henan Key Laboratory of Plant Stress Biology,

College of Life Sciences, Henan University, Kaifeng 475004, China

2 Henan University of Animal Husbandry and Economy, Zhengzhou 450011, China

ABSTRACT

Calcineurin B-like (CBL) proteins, as calcium sensors, play pivotal roles in plant responses to diverse abiotic stresses and in growth and development through interaction with CBL-interacting protein kinases (CIPKs). However, knowledge about functions and evolution of CBLs in Gossypium plants is scarce. Here, we conducted a genome-wide survey and identified 13, 13 and 22 CBL genes in the progenitor diploid Gossypium arboreum and Gossypium raimondii, and the cultivated allotetraploid Gossypium hirsutum, respectively. Analysis of physical properties, chromosomal locations, conserved domains and phylogeny indicated rather conserved nature of CBLs among the three Gossypium species. Moreover, these CBLs have closer genetic evolutionary relationship with the CBLs from cocoa than with those from other plants. Most CBL genes underwent evolution under purifying selection in the 3 Gossypium plants. Additionally, nearly all G. hirsutum CBL (GhCBL) genes were expressed in the root, stem, leaf, flower and fiber. Many GhCBLs were preferentially expressed in the flower while several $G h C B L s$ were mainly expressed in roots. Expression patterns of GhCBL genes in response to potassium deficiency were also studied. The expression of most GhCBLs were moderately induced in roots after treatments with low-potassium stress. Yeast two-hybrid experiments indicated that GhCBL1-2, GhCBL1-3, GhCBL4-4, GhCBL8, GhCBL9 and GhCBL10-3 interacted with GhCIPK23, respectively. Our results provided a comprehensive view of the CBLs and valuable information for researchers to further investigate the roles and functional mechanisms of the CBLs in Gossypium. 
39 Keywords Gossypium; calcineurin B-like proteins (CBLs); gene family; phylogeny; gene

40

expression

\section{INTRODUCTION}

Calcium ion $\left(\mathrm{Ca}^{2+}\right)$ plays pivotal roles in mediating and regulating many fundamental growth and developmental processes and in response to various environmental stimuli (Luan, 2009; Kudla et al., 2010; Sarwat et al., 2013). The $\mathrm{Ca}^{2+}$ signals are primarily perceived by some $\mathrm{Ca}^{2+}$ sensors including $\mathrm{Ca}^{2+}$ dependent protein kinases, calmodulins and calcineurin B-like proteins (CBLs), and then are transmitted by these sensors to downstream targets to initiate diverse cellular responses (Luan, 2009; Kudla, et al., 2010; Sarwat et al., 2013).

CBLs are proteins sharing sequence similarity with the B subunit of calcineurin B in yeast and neuronal calcium sensors in animals (Kudla et al., 1999). Each CBL has at least three EF domains and $\mathrm{Ca}^{2+}$-binding sites (Mohanta et al., 2015; Mao et al., 2016). CBLs relay $\mathrm{Ca}^{2+}$ signals through interaction with and activation of the CBL-interacting protein kinases (CIPKs). Moreover, CBL-CIPK has been demonstrated to serve as an essential signaling network regulating plant responses to multiple abiotic stresses such as salinity, $\mathrm{K}^{+}$deficiency, excess of $\mathrm{Mg}^{2+}$ and drought (Sanyal et al., 2015; Thoday-Kennedy et al., 2015; Mao et al., 2016). It also modulates growth and development, absorption and/or transport of nitrate, ammonium and iron, sustaining of $\mathrm{H}^{+}$homeostasis, and transduction of reactive oxygen species signals in plants (Sanyal et al., 2015; Thoday-Kennedy et al., 2015; Mao et al., 2016).

In Arabidopsis, 10 genes (CBL1-10) encoding CBL proteins have been found (Kolukisaoglu et al., 2004). $C B L 1$ and $C B L 9$ were reported to positively regulate the uptake and transport of $\mathrm{K}^{+}$, $\mathrm{NO}_{3}{ }^{-}, \mathrm{NH}_{4}{ }^{+}$, aluminum and iron, and the promotion of stomatal opening (Li et al., 2006; Xu et al., 2006; Ho et al., 2009; Mao et al., 2016; Tian et al., 2016; Ligaba-Osena et al., 2017; Straub et al., 2017). $C B L 1$ and $C B L 9$ also affect abscisic acid (ABA)-induced stomatal closure and ROS signaling (Pandey et al., 2004; Cheong et al., 2007; Drerup et al., 2013). CBL2 plays a negative role in the activation of plasma membrane (PM) $\mathrm{H}^{+}$-ATPase (Fuglsang et al., 2007). Moreover, 
$C B L 2$ and $C B L 3$ are cooperatively implicated in sequestering $\mathrm{Mg}^{2+}$ and modulation of pollen germination and tube growth (Steinhorst et al., 2015; Tang et al., 2015). CBL3 are also engaged in $\mathrm{K}^{+}$distribution and translocation (Liu et al., 2013). CBL4 was proven to be a crucial regulator for excluding $\mathrm{Na}^{+}$and translocation of AKT2 (Arabidopsis $\mathrm{K}^{+}$transporter 2) from endoplasmic reticulum to PM (Held et al., 2011). CBL10 is involved in enhancing salt tolerance, stimulating $\mathrm{K}^{+}$absorption, and modulating GTPase activity (Kim et al., 2007; Ren et al., 2013; Cho et al., 2016). In cotton (Gossypium hirsutum), GhCBL2 and GhCBL3 appear to modulate fiber elongation (Gao et al., 2008). Many $C B L s$ in other plant species also play important parts in regulating the responses to various abiotic stress as well as growth and development (Li et al., 2014a; Thoday-Kennedy et al., 2015).

In recent years, multiple CBL gene families have been identified at genome-wide levels in rice, maize, wheat and other plants (Kolukisaoglu et al., 2004; Zhang et al., 2014; Sun et al., 2015; Li et al., 2016; Zhang et al., 2016). Some conserved domains such as EF-hands, myristoylation and palmitoylation sites were discovered in CBLs (Kolukisaoglu et al., 2004; Mohanta et al., 2015). The expression patterns of many $C B L$ genes were also investigated in different tissues and in response to various abiotic stresses in plants (Mohanta et al., 2015; Zhang et al., 2016). These findings lay the foundation for people to further explore the functional mechanisms of CBLs in plants. However, to date, knowledge about genomics and evolutionary information of CBLs in Gossypium is limited.

Cotton is an essential tetraploid fiber crop that supplies lint for the textile industry worldwide. It is considered to descend from an ancestral combination of two diploid most similar to modern A (for example Gossypium arboretum) and D genome species (Gossypium raimondii) (Wendel et al., 2010).

Cotton growth and development are severely threatened by diverse abiotic stresses such as drought, salinity and potassium starvation (Allen, 2000). Therefore, enhancing stress tolerance of cotton cultivars is one of most important strategies for us to improve their productivity and quality. Potassium is a vital macronutrient for plants, especially for cotton. Potassium shortage in 
92

93

soil seriously affects the yield and quality of cotton (Oosterhuis et al., 2013). Moreover, it has been demonstrated that $\mathrm{K}^{+}$uptake is controlled by CBLs through interacting with CIPK23 in Arabidopsis and rice under potassium deficiency (Li et al., 2014a; Mao et al., 2016). Research is needed to determine which and how CBLs modulate $\mathrm{K}^{+}$absorption in cotton. In this report, genome-wide and comprehensive analyses of the CBL family in G. arboreum, G. raimondii and G. hirsutum were conducted. The expression patterns of GhCBLs were monitored in tissues and in response to potassium deficiency in cotton. These analyses will provide a basis for further investigation of the functions of CBLs in Gossypium.

\section{MATERIALS AND METHODS}

\section{Identification of CBL family in Gossypium}

The genome sequences of $G$. arboreum (BGI-CGB v2.0 assembly genome), G. raimondii (JGI assembly v2.0 data.) and G. hirsutum (NAU-NBI v1.1 assembly genome) were downloaded from the CottonGen database (www.cottongen.org), respectively. The protein sequences of 10 Arabidopsis CBLs were applied as queries to search the three genomes using BLAST-2.4.0 software (ftp://ftp.ncbi.nlm.nih.gov/blast/executables/blast+/LATEST) with default parameters $\left(\right.$ E-value $\left.<\mathrm{e}^{-10}\right)$. EF-hand domains, the typical CBL domains, were analyzed within the candidate CBLs one by one using online software SMART (http://smart.embl-heidelberg.de/). The CBL motifs were also queried against the Pfam databases (Finn et al., 2010). The putative CBLs with questionable annotations (i.e. having a typical CBL domain but low E-value or low coverage of a domain) were manually reanalyzed.

\section{Analysis of Gossypium CBLs family}

The properties of the Gossypium CBL proteins were analyzed using online tools ExPaSy (http://web.expasy.org/protparam/). The subcellular localizations of the CBLs were examined in the website http://www.csbio.sjtu.edu.cn/bioinf/Cell-PLoc/. The locations of the CBLs in chromosomes were assessed by MapInspect software (http://www.softsea.com/review/MapInspect.html). Structures of the CBLs were determined by 
118 GSDS (http://gsds.cbi.pku.edu.cn/). The conserved domains in the CBLs were affirmed by

119 SMART (http://smart.embl-heidelberg.de). The sequence logo of myristoylation motif in the

120 CBLs were generated by MEME program (http://meme-suite.org/tools/meme).

121 Analyses of synteny and $\mathrm{Ka} / \mathrm{Ks}$ ratio

122 The homologous gene pairs among the Gossypium CBLs were searched by the MCScanx 123 software (http://chibba.pgml.uga.edu/mcscan2/). The gene collinearity results were obtained by

124 CIRCOS program (http://www.circos.ca/). The ratio of Ka (nonsynonymous substitution rate) to

125 Ks (synonymous substitution rate) of the CBL genes were estimated by PAML program 126 (http://abacus.gene.ucl.ac.uk/software/paml.html).

127 Phylogenetic analysis of CBLs

128 The CBL data were downloaded from the websites for various plant species including

129 Arabidopsis thaliana (http://www.arabidopsis.org/), Oryza sativa (http://rapdb.dna.affrc.go.jp),

130 Vitis vinifera (http://www.genoscope.cns.fr/spip/Vitis-vinifera-e.html), Populus trichocarpa

131 (http://www.phytozome.net/poplar), Glycine max (http://www.phytozome.net/soybean),

132 Theobroma cacao (http://cocoagendb.cirad.fr), Carica papaya (http://asgpb.mhpcc.hawaii.edu)

133 and castor bean (http://castorbean.jcvi.org). The full-length amino acid sequences of CBL

134 proteins were aligned using Clustal W software through pairwise and multiple alignment with

135 default parameters (Larkin et al, 2007). Then, phylogenetic trees were generated based on the

136 alignment results using the neighbor joining method (Neighbor-Joining, NJ) and 1,000 bootstrap

137 trials with the MEGA 5.0 software (http://www.megasoftware.net/).

138 Expression analysis of $G \boldsymbol{C} C B L$ genes in tissues and in response to potassium deficiency

139 For measuring the expression of the GhCBLs in tissues, samples of roots, stems and leaves were 140 collected from 20-day-old G. hirsutum TM-1 plants normally grown in soil containing 1:1 (v:v)

141 peat:vermiculite in a growth chamber (day/night temperature cycle of $28^{\circ} \mathrm{C} / 26^{\circ} \mathrm{C}, 14 \mathrm{~h} \mathrm{light} / 10 \mathrm{~h}$ 142 dark, and about 50\% relative humidity). Flowers were isolated in the morning at the first day of 143 anthesis from cotton grown in the field. The fibers at elongation stage were obtained from the 144 ovules (23 days post anthesis). For monitoring the expression of GhCBLs in responding to 
145 potassium deprivation, cotton plants grew in clean small pebbles (watered by liquid 1/2 MS

146 medium) (Murashige and Skoog, 1962) in the growth chamber described above for 3 weeks.

147 Then, the plants were watered with $\mathrm{K}^{+}$-lacking liquid $1 / 2 \mathrm{MS}$ medium $\left(\mathrm{KNO}_{3}\right.$ was replaced by

$148 \mathrm{NH}_{4} \mathrm{NO}_{3}$ and $\mathrm{KH}_{2} \mathrm{PO}_{4}$ was replaced by $\mathrm{NH}_{4} \mathrm{H}_{2} \mathrm{PO}_{4}$ ) for $0 \mathrm{~h}, 6 \mathrm{~h}, 2 \mathrm{~d}$ and $5 \mathrm{~d}$, respectively.

149 Meanwhile, some $\mathrm{K}^{+}$-starved seedlings for $5 \mathrm{~d}$ were resupplied with $\mathrm{K}^{+}$(watered with $\mathrm{K}^{+}$-

150 contained 1/2 MS medium) for $3 \mathrm{~h}$. The cotton roots were collected, immediately frozen in liquid

151 nitrogen and stored at $-70^{\circ} \mathrm{C}$. Total RNA of samples was extracted using RNA Pure Plant Kit's

152 protocol (TIANGEN Company). The purity of RNA was examined using a Nanodrop2000

153 nucleic acid analyzer. The A260/280 ratio for each RNA sample was about 2.0. Then, total

154 cDNA was synthesized using M-MLV reserve transcriptase synthesis system (Promega, USA)

155 following the instructions in the Promega kit

156 (https://tools.thermofisher.com/content/sfs/manuals/superscriptIII_man.pdf.)

157 Quantitative real-time RT-PCR (qRT-PCR) experiments were performed using the cDNA,

158 SYBR Green Master mix, the specific primers of $G h C B L$ genes (Table 1), and an ABI 7500 real-

159 time PCR system. GhUBQ7 was used as the internal control. At least three biological replicates

160 were carried out.

161 Table 1 Gene primers used for quantitative real-time RT-PCR experiments

\begin{tabular}{|c|c|c|c|}
\hline Genes & AGI number & Forward primers $\left(5^{\prime}-3^{\prime}\right)$ & Reverse primers $\left(5^{\prime}-3^{\prime}\right)$ \\
\hline GhUBQ7 & Gh_A11G0969 & $\begin{array}{l}\text { GAAGGCATTCCACCTGA } \\
\text { CCAAC }\end{array}$ & $\begin{array}{l}\text { CTTGACCTTCTTCTTCTTG } \\
\text { TGCTTG }\end{array}$ \\
\hline GhCBL1-1 & Gh_A11G0257 & $\begin{array}{l}\text { GAGCGTAACGAGGTCAA } \\
\text { GCAAA }\end{array}$ & $\begin{array}{l}\text { CTTCCCGTCCTGATTAATG } \\
\text { TCC }\end{array}$ \\
\hline GhCBL1-2 & Gh_D11G0276 & $\begin{array}{l}\text { TTTTGTTCGAGCACTCAA } \\
\text { TGTTT }\end{array}$ & $\begin{array}{l}\text { TTGCCTCAATCGTTTCATC } \\
\text { AG }\end{array}$ \\
\hline GhCBL1-3 & Gh_A03G0043 & $\begin{array}{l}\text { GACATTCTTGGAAGCCG } \\
\text { ATA }\end{array}$ & $\begin{array}{l}\text { CTGAGGTATGGGAGGGTC } \\
\text { AT }\end{array}$ \\
\hline GhCBL1-4 & Gh_D09G1875 & $\begin{array}{l}\text { AGAGTAATGACCCTCCC } \\
\text { ATACCTAA }\end{array}$ & $\begin{array}{l}\text { CGAGCGAGTATTCTCCGA } \\
\text { CAA }\end{array}$ \\
\hline GhCBL1-5 & Gh_A09G1766 & $\begin{array}{l}\text { GGATGCCGACACTAACC } \\
\text { AGG }\end{array}$ & TCCAACAACGTAGCGGCC \\
\hline GhCBL3-1 & Gh_A01G0740 & $\begin{array}{l}\text { AGTTTGCTCGTGCTCTCT } \\
\text { CTGT }\end{array}$ & $\begin{array}{l}\text { ATCATCTGAAAGGTTCAT } \\
\text { GCCA }\end{array}$ \\
\hline GhCBL3-2 & Gh_D01G0760 & GCAAGAGAGACCGTTTT & AATCTTATCGTCAATGGG \\
\hline
\end{tabular}




\begin{tabular}{|c|c|c|c|}
\hline & & TAGTG & CG \\
\hline \multirow{2}{*}{$G h C B L 3-3$} & \multirow{2}{*}{ Gh_A13G1099 } & GGGCTGATTAACAAGGA & ACAGAAAGAGCACGAGC \\
\hline & & GGAGT & AAACT \\
\hline \multirow{2}{*}{ GhCBL3-4 } & \multirow{2}{*}{ Gh_D13G1364 } & ATGGGCTGATTAACAAG & GACAGAAAGAGCACGAG \\
\hline & & GAGGAG & CGAAC \\
\hline \multirow{2}{*}{$G h C B L 3-5$} & \multirow{2}{*}{ Gh_A04G0051 } & GCGGTGATAGATGACGG & GACAGAGAGAGCACGAG \\
\hline & & $\mathrm{ACT}$ & CAA \\
\hline \multirow{2}{*}{ GhCBL3-6 } & \multirow{2}{*}{ Gh_D05G3682 } & TACACGCTTCCGACCCT & ATCAATGAGCCCGTCGTA \\
\hline & & ATT & $\mathrm{AC}$ \\
\hline \multirow{2}{*}{ GhCBL4-1 } & \multirow{2}{*}{ Gh_A11G0126 } & ACGGCTAGTGAAGTAGA & CGAACAAATCAAAAACCC \\
\hline & & ATCCC & TGTC \\
\hline \multirow{2}{*}{ GhCBL4-2 } & \multirow{2}{*}{ Gh_D11G0140 } & TTCTTGCTGCTGAAACAC & CGAACAAATCAAAAACCC \\
\hline & & $\mathrm{CT}$ & TG \\
\hline \multirow{2}{*}{ GhCBL4-3 } & \multirow{2}{*}{ Gh_A12G2144 } & TAAGCGTCTTTCATCCCA & TGATTCACCAAGCAGAGC \\
\hline & & $\mathrm{AC}$ & $\mathrm{CA}$ \\
\hline \multirow{2}{*}{ GhCBL4-4 } & \multirow{2}{*}{ Gh_A09G1696 } & AACTTAGACACAAGGCT & GAGGTTCTGCTTATTGCTG \\
\hline & & GGGTATG & TTTTT \\
\hline \multirow{2}{*}{ GhCBL4-5 } & \multirow{2}{*}{ Gh_D12G2320 } & CCTGAGGAGGTCAAGGA & AAATTGGGTTGCGAGCTA \\
\hline & & GATG & CAAA \\
\hline \multirow{2}{*}{ GhCBL9 } & \multirow{2}{*}{ Gh_D08G1764 } & GACATTCTTGGATGCCG & ACGCAGCAACCTCGTCTA \\
\hline & & ACA & CT \\
\hline \multirow{2}{*}{ GhCBL10-1 } & \multirow{2}{*}{ Gh_A06G0800 } & AGTCTCACAGTGGCGGC & TTCATTGGCAAGACGGGT \\
\hline & & A & AA \\
\hline \multirow{2}{*}{ GhCBL10-2 } & \multirow{2}{*}{ Gh_D06G0922 } & GTCGCGAGAAATGCCGT & ATTCTCGCCGTATGGAGT \\
\hline & & TAT & TTG \\
\hline \multirow{2}{*}{ GhCBL10-3 } & \multirow{2}{*}{ Gh_A05G0335 } & CTGAAATGAATTTGTCC & ACTGGAAATAGTAGTTCA \\
\hline & & GATGAC & TCACGGA \\
\hline \multirow{2}{*}{ GhCBL10-4 } & \multirow{2}{*}{ Gh_D05G0440 } & TCTGGAATGAATTTGTC & CTGGAAATAGGAGTTCTT \\
\hline & & GGATG & CACGG \\
\hline
\end{tabular}

162 Yeast two-hybrid (Y2H) analysis

163 The full-length CDS sequences of GhCBLs and GhCIPK23 genes were amplified, sequenced and 164 cloned into pGBKT7 and pGADT7 vectors, respectively, using primers listed in Table 2. The 165 plasmids were then transformed into yeast strain AH109 according to the method described in 166 page 18-21 in Yeast Protocols Handbook (Clontech,

167 http://www.clontech.com/xxclt_searchResults.jsp). The cotransformants were plated on non168 selective SD/-Leu/-Trp (synthetic dropout medium without Leu and Trp) solid medium and 169 selective SD/-Leu/-Trp/-His/-Ade solid medium. The medium was prepared by ourselves. The 170 concentrations of each component for SD/-Leu/-Trp medium are as follows: L-isoleucine 300 171 mg/L, L-valine 1.5 g/L, adenine 200 mg/L, L-arginine 200 mg/L, L-lysine 300 mg/L, L- 
172 methionine $200 \mathrm{mg} / \mathrm{L}$, L-phenylalanine $500 \mathrm{mg} / \mathrm{L}$, L-threonine $2 \mathrm{~g} / \mathrm{L}$, L-tyrosine $300 \mathrm{mg} / \mathrm{L}$, L-

173 histidine $200 \mathrm{mg} / \mathrm{L}$, uracil $200 \mathrm{mg} / \mathrm{L}$, yeast nitrogen base without amino acids $6.7 \mathrm{~g} / \mathrm{L}$, glucose 20

$174 \mathrm{~g} / \mathrm{L}$. Serial 1:10 dilutions of the cotransformants were made in water, and $2 \mu 1$ of the dilution was

175 dropped to generate one spot. Plates were incubated at $30^{\circ} \mathrm{C}$ for $3-4 \mathrm{~d}$. 5-bromo-4-chloro-3-

176 indoxyl- $\alpha-\mathrm{D}$-galactopyranoside (X- $\alpha$-Gal) staining assay was carried out following the

177 instruction (the Clontech protocol, page 26).

178 Table 2 Gene primers used for yeast two-hybrid experiments

\begin{tabular}{clll}
\hline Genes & AGI number & Forward primers (5'-3') & \multicolumn{1}{c}{ Reverse primers (5'-3') } \\
\hline GhCBL1-2(BD) & Gh_D11G0276 & CCGGAATTCATGGGCTG & CGCGGATCCTGTGGCAAC \\
& & CTTTCAATCT & CTCATCA \\
GhCBL1-3(BD) & Gh_A03G0043 & CCGGAATTCATGGGTTG & CGCGGATCCAGTGGCAAC \\
& & CTTTCATTCT & TTCATCTAC \\
GhCBL1-4(BD) & Gh_D09G1875 & CGCGGATCCATGGGCTG & GCACTGCAGTATGCCATT \\
& & CTTGCAATGTAAA & CGCCGAGCGAGT \\
GhCBL1-5(BD) & Gh_A09G1766 & ATAGGATCCATGGGCTG & GCACTGCAGGTATAACAT \\
& & CTTGCAATGTA & CGGTATTATGTACCT \\
GhCBL3-2(BD) & Gh_D01G0760 & CGCGGATCCATGTTGCA & GCACTGCAGTGTATCATC \\
& & GTGCATAGAC & AACTTGAGAGTGGAAAA \\
GhCBL3-4(BD) & Gh_D13G1364 & CGCGGATCCATGGGAAT & GCACTGCAGTTTGCCACC \\
& & TTGTTGTTT & CATATTCAACT \\
GhCBL4-1(BD) & Gh_A11G0126 & CGCGGATCCATGAAATG & GCACTGCAGATCTCCATT \\
& & GTGTTTCAAACT & GACGGAGACGCT \\
GhCBL4-3(BD) & Gh_A12G2144 & CGCGGATCCATGGGTTG & GCACTGCAGCTTATTCCC \\
& & TTTTTGCTTG & AACGATTTCAGCT \\
GhCBL4-4(BD) & Gh_A09G1696 & CGCGGATCCATGGGCTG & GCACTGCAGGTTTTTCTC \\
& & CTTTTGCTTG & AATTCTTCACTGGT \\
GhCBL8(BD) & Gh_D09G1801 & CGCGGATCCATGGGCTG & GCACTGCAGATTCTTCAC \\
& & CTTTTGCTTGAAGAA & TGGTTGCTGCAAATCTGA \\
& & & GAC \\
GhCBL9(BD) & Gh_D08G1764 & CCGGAATTCATGGGCTG & CGCGGATCCCGCAGCAAC \\
& & CTTTCATTCT & CTCGTCTA \\
$G h C B L 10-3(B D)$ & Gh_A05G0335 & CGCGGATCCATGGATTC & GCACTGCAGCCGGAGATA \\
& & AACTAGCAAAACC & GGAAAGGGCCAA \\
GhCIPK23(AD) & Gh_A06G1219 & CCGGAATTCATGGCGAA & CGCGGATCCACCATCCTT \\
& & TCGCACTAGT & TTCTTCCAC \\
\hline
\end{tabular}

179 RESULTS 
180

181

182

183

184

185

186

187

188

189

190

191

192

193

194

195

196

197

198

199

200

201

202

203

204

Genome-wide identification of the CBL family in two progenitor diploid and the tetraploid cotton species

The CBL genes in Gossypium were identified using the homologous alignment method. A total of 13, 13, and $22 \mathrm{CBL}$ genes were respectively detected in A genome (G. arboretum), D genome (G. raimondii) and $\mathrm{A}_{\mathrm{t}} \mathrm{D}_{\mathrm{t}}$ genome $(G$. hirsutum) using 10 Arabidopsis $\mathrm{CBL}$ protein sequences as queries (Table 3). Further, the CBL candidate genes in Gossypium were confirmed by domain analysis programs of Pfam and SMART. The CBL family members were named according to their orthologous similarity to the 10 Arabidopsis CBL proteins (Mohanta et al., 2015). In general, the CBLs in G. arboretum, G. raimondii and G. hirsutum were named GaCBLs, GrCBLs and $G h C B L s$, respectively.

Most CBLs had very similar physical properties in the 3 Gossypium plants (Table 3). The open reading frame (ORF) lengths of the $\mathrm{CBL}$ genes ranged from $570 \mathrm{bp}$ to $882 \mathrm{bp}$ except that of GhCBL3-6, whose ORF length was 3981 bp. The GaCBL and GrCBL proteins contained 199279 and 209-253 amino acids (AA), respectively, while GhCBLs were composed of 189-293 AA except GhCBL3-6, which consisted of 1326 AA. The molecular weights (MWs) of GaCBLs varied from $23.25 \mathrm{kDa}$ (GaCBL10-1) to $32.43 \mathrm{kDa}$ (GaCBL10-2), and of GrCBLs ranged from $23.25 \mathrm{kDa}(\mathrm{GrCBL} 3-3)$ to $29.26 \mathrm{kDa}$ (GrCBL10-1). The sizes of GhCBLs were 21.64 kDa (GhCBL3-4) to $33.56 \mathrm{kDa}$ (GhCBL10-1) with an exception of GhCBL3-6 (150.21 kDa). The theoretical isoelectric point ( $\mathrm{pI})$ is small for overwhelming majority of the CBLs, ranging from 4.65 (GaCBL9) to 5.64 (GhCBL4-5). By contrast, pI of GhCBL3-6 was 8.05 (Table 3).

Putative subcellular localizations of the Gossypium CBL proteins were also analyzed. It was predicted that all of CBLs were located in cell membrane except that GhCBL3-6 was in the nucleus (Table 3). The quite different characteristics of GhCBL3-6 from other members suggest that GhCBL3-6 likely play a special role in cotton.

Table 3 The CBL family genes in Gossypium

\begin{tabular}{|c|c|c|c|c|c|c|c|}
\hline Gene name & Gene ID & $\mathrm{pI}$ & $\begin{array}{l}\text { MW } \\
(\mathrm{kDa})\end{array}$ & $\begin{array}{l}\text { Hydro- } \\
\text { philicity }\end{array}$ & $\begin{array}{l}\text { Predicted } \\
\text { subcellular } \\
\text { localization }\end{array}$ & $\begin{array}{l}\text { amino } \\
\text { acid } \\
\text { residues }\end{array}$ & $\begin{array}{l}\text { coding } \\
\text { sequence }\end{array}$ \\
\hline
\end{tabular}




\begin{tabular}{|c|c|c|c|c|c|c|c|}
\hline GaCBL1-1 & Cotton_A_16036 & 4.74 & 24.33 & -0.163 & Cell membrane & 213 & 642 \\
\hline GaCBL1-2 & Cotton_A_16034 & 4.74 & 24.33 & -0.163 & Cell membrane & 213 & 642 \\
\hline GaCBL1-3 & Cotton_A_16590 & 5.06 & 25.39 & -0.216 & Cell membrane & 221 & 666 \\
\hline GaCBL1-4 & Cotton_A_09151 & 4.72 & 24.39 & -0.142 & Cell membrane & 213 & 642 \\
\hline GaCBL2 & Cotton_A_07469 & 4.78 & 25.94 & -0.2 & Cell membrane & 226 & 681 \\
\hline GaCBL3-1 & Cotton_A_06492 & 4.77 & 25.98 & -0.189 & Cell membrane & 226 & 681 \\
\hline GaCBL3-2 & Cotton_A_02147 & 5.08 & 27.68 & -0.314 & Cell membrane & 240 & 723 \\
\hline GaCBL4-1 & Cotton_A_02388 & 4.81 & 24.88 & -0.13 & Cell membrane & 220 & 663 \\
\hline GaCBL4-2 & Cotton_A_13237 & 4.97 & 24.47 & -0.173 & Cell membrane & 215 & 648 \\
\hline GaCBL8 & Cotton_A_08153 & 4.89 & 23.48 & -0.134 & Cell membrane & 205 & 618 \\
\hline GaCBL9 & Cotton_A_13238 & 4.65 & 24.22 & -0.141 & Cell membrane & 210 & 633 \\
\hline GaCBL10-1 & Cotton_A_14000 & 4.55 & 23.25 & -0.175 & Cell membrane & 199 & 600 \\
\hline GaCBL10-2 & Cotton_A_34841 & 4.82 & 32.43 & -0.028 & Cell membrane & 279 & 840 \\
\hline GrCBL1-1 & Gorai.007G030300 & 4.72 & 24.38 & -0.143 & Cell membrane & 213 & 642 \\
\hline GrCBL1-2 & Gorai.003G178700 & 4.71 & 24.45 & 0.075 & Cell membrane & 214 & 645 \\
\hline GrCBL1-3 & Gorai.004G191400 & 4.67 & 23.86 & 0.016 & Cell membrane & 209 & 630 \\
\hline GrCBL1-4 & Gorai.006G214700 & 4.99 & 25.39 & -0.226 & Cell membrane & 221 & 666 \\
\hline GrCBL3-1 & Gorai.013G150400 & 4.79 & 25.96 & -0.208 & Cell membrane & 226 & 681 \\
\hline GrCBL3-2 & Gorai.002G102900 & 4.77 & 25.98 & -0.189 & Cell membrane & 226 & 681 \\
\hline GrCBL3-3 & Gorai.009G450400 & 4.84 & 23.25 & -0.21 & Cell membrane & 226 & 681 \\
\hline GrCBL4-1 & Gorai.007G015400 & 4.78 & 24.91 & -0.193 & Cell membrane & 233 & 702 \\
\hline GrCBL4-2 & Gorai.006G207100 & 4.98 & 25.26 & -0.161 & Cell membrane & 221 & 666 \\
\hline GrCBL4-3 & Gorai.008G255900 & 5.11 & 24.02 & -0.161 & Cell membrane & 211 & 636 \\
\hline GrCBL9 & Gorai.008G255800 & 4.66 & 24.58 & -0.139 & Cell membrane & 213 & 642 \\
\hline GrCBL10-1 & Gorai.010G101400 & 4.74 & 29.26 & -0.096 & Cell membrane & 252 & 759 \\
\hline GrCBL10-2 & Gorai.009G045600 & 4.83 & 29.23 & -0.095 & Cell membrane & 253 & 762 \\
\hline GhCBL1-1 & Gh_A11G0257 & 4.72 & 24.44 & -0.148 & Cell membrane & 213 & 642 \\
\hline GhCBL1-2 & Gh_D11G0276 & 4.79 & 24.38 & -0.145 & Cell membrane & 213 & 642 \\
\hline GhCBL1-3 & Gh_A03G0043 & 4.98 & 22.76 & -0.163 & Cell membrane & 199 & 600 \\
\hline GhCBL1-4 & Gh_D09G1875 & 5.06 & 25.69 & -0.194 & Cell membrane & 224 & 675 \\
\hline GhCBL1-5 & Gh_A09G1766 & 5.51 & 23.23 & -0.165 & Cell membrane & 200 & 603 \\
\hline GhCBL3-1 & Gh_A01G0740 & 4.77 & 25.98 & -0.189 & Cell membrane & 226 & 681 \\
\hline GhCBL3-2 & Gh_D01G0760 & 4.77 & 25.99 & -0.189 & Cell membrane & 226 & 681 \\
\hline GhCBL3-3 & Gh_A13G1099 & 4.84 & 23.25 & -0.21 & Cell membrane & 202 & 609 \\
\hline GhCBL3-4 & Gh_D13G1364 & 4.98 & 21.64 & -0.205 & Cell membrane & 189 & 570 \\
\hline GhCBL3-5 & Gh_A04G0051 & 5.14 & 21.76 & -0.274 & Cell membrane & 189 & 570 \\
\hline GhCBL3-6 & Gh_D05G3682 & 8.05 & 150.21 & -0.284 & Nucleus & 1326 & 3981 \\
\hline
\end{tabular}




\begin{tabular}{llllllll}
\hline GhCBL4-1 & Gh_A11G0126 & 4.77 & 23.01 & -0.059 & Cell membrane & 201 & 606 \\
GhCBL4-2 & Gh_D11G0140 & 4.82 & 24.97 & -0.185 & Cell membrane & 220 & 663 \\
GhCBL4-3 & Gh_A12G2144 & 4.97 & 24.5 & -0.175 & Cell membrane & 215 & 648 \\
GhCBL4-4 & Gh_A09G1696 & 5.27 & 28.4 & -0.184 & Cell membrane & 248 & 747 \\
GhCBL4-5 & Gh_D12G2320 & 5.64 & 25.06 & 0.023 & Cell membrane & 218 & 657 \\
GhCBL8 & Gh_D09G1801 & 4.85 & 24.74 & -0.177 & Cell membrane & 217 & 654 \\
GhCBL9 & Gh_D08G1764 & 4.74 & 23.8 & -0.032 & Cell membrane & 209 & 630 \\
GhCBL10-1 Gh_A06G0800 & 5.18 & 33.56 & -0.143 & Cell membrane & 293 & 882 \\
GhCBL10-2 Gh_D06G0922 & 4.95 & 30.41 & -0.159 & Cell membrane & 265 & 798 \\
GhCBL10-3 Gh_A05G0335 & 5.16 & 30.48 & -0.114 & Cell membrane & 262 & 789 \\
GhCBL10-4 Gh_D05G0440 & 5.01 & 30.25 & -0.08 & Cell membrane & 262 & 789 \\
\hline
\end{tabular}

205 Distribution of the Gossypium CBL family members in the whole genome

206 Chromosomal distributions of the $C B L$ genes were examined in Gossypium. In general, the CBLs

207 were unevenly distributed among the Gossypium chromosomes. Thirteen GaCBLs were 208 distributed on 7 chromosomes. Among them, 3 GaCBLs were located on each of Gachr07 and 209 Gachr11 chromosomes. Two GaCBLs were situated in each of Gachr06 and Gachr13, and 1 $210 G a C B L$ was on Gachr01, Gachr08 and Gachr09, respectively (Fig.1). Thirteen GrCBL genes 211 were identified on 9 chromosomes. Each of the 4 chromosomes Grchr06, Grchr07, Grchr08 and 212 Grchr09 owned 2 genes, and other chromosomes (Grchr02, Grchr03, Grchr04, Grchr10, Grchr13) 213 individually contained 1 gene (Fig.1). Likewise, 22 GhCBL family members were mapped onto 21417 chromosomes. Each of the 5 chromosomes Ghchr09, Ghchr11, Ghchr19, Ghchr21 and 215 Ghchr23 had $2 C B L$ members, and other chromosomes individually carried $1 C B L$ member 216 (Fig.1). We observed the phenomena of 2 CBL genes joining together in a chromosome. For 217 instance, GaCBL4-2 and GaCBL9 were mapped within 16.0 Mb in Gachr06, and GrCBL4-3 and 218 GrCBL9 were mapped within $53.8 \mathrm{Mb}$ in Grchr08. These findings suggest that tandem 219 duplication play a role in generating these genes during evolution. 


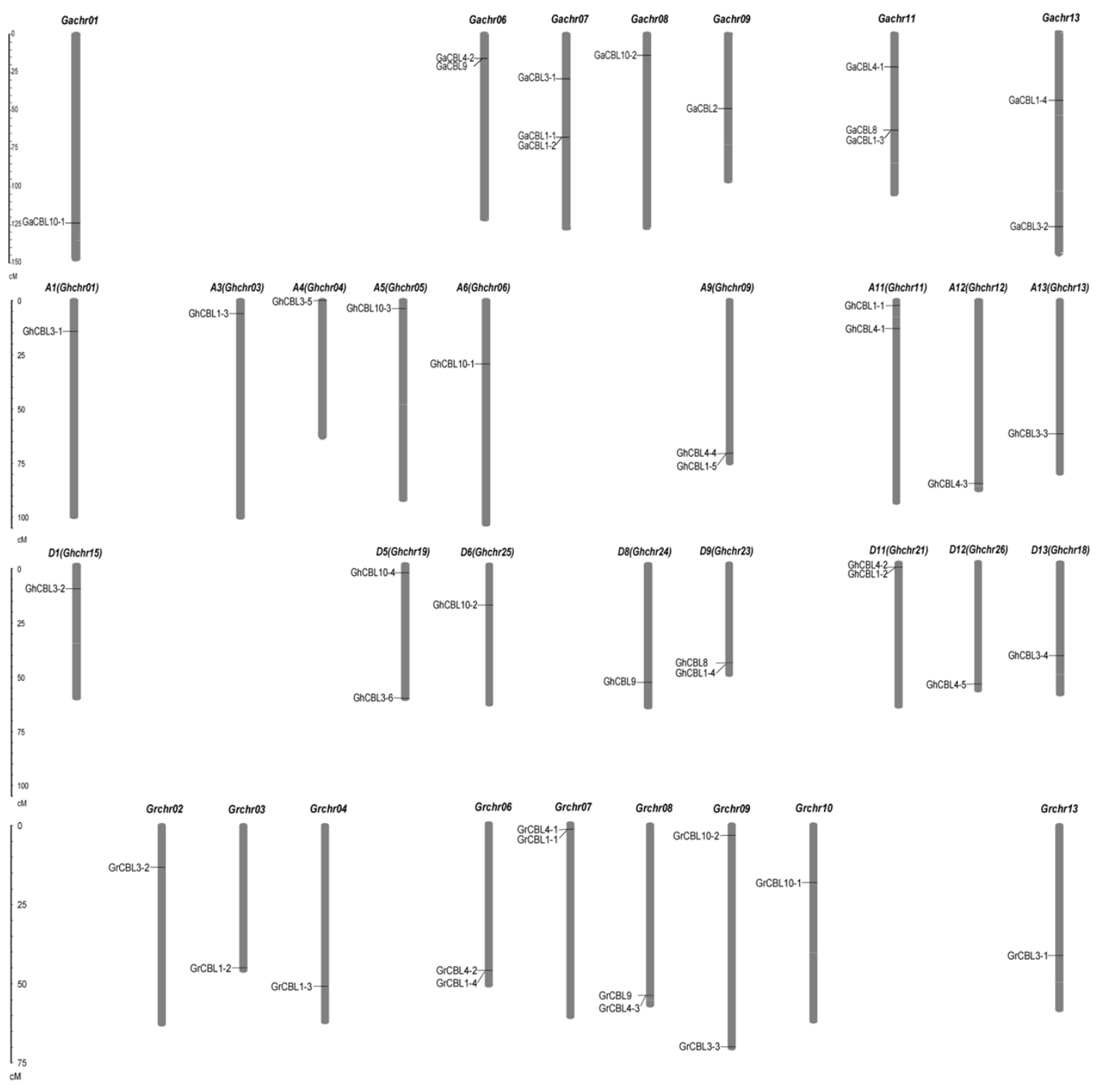

Fig.1 Distributions of the $C B L$ family genes on chromosomes in Gossypium

The GaCBLs, GrCBLs and GhCBLs are from G. arboreum, G. raimondii and G. hirsutum, respectively.

\section{Phylogenetic analysis and structural properties of $C B L$ genes in Gossypium}

221 To determine the sequence similarity relationship of the CBLs among G. arboreum, G. raimondii,

222 and $G$. hirsutum, the phylogenetic tree for the 48 CBLs was constructed. The CBLs can be

223 classified into four families (I to IV) (Fig.2a). Family I consisted of 12 CBLs (3 GaCBLs, 3

224 GrCBLs and 6 GhCBLs). The members in family II were 8 CBLs (2 GaCBLs, 2 GrCBLs and 4

225 GhCBLs). Family III contained 14 CBLs (4 GaCBLs, 4 GrCBLs and 6 GhCBLs). Family IV had 
226

227

228

229

230

231

232

233

234

235

236

237

238

239

14 CBLs (4 GaCBLs, 4 GrCBLs and 6 GhCBLs) (Fig.2a).

The structure of a protein is closely related to its functions in cells. We therefore identified the intron-exon structures of the $C B L$ genes in Gossypium by mapping the cDNA sequences onto their genomic sequences. Most of $G a C B L s$ and $G r C B L s$ owned 8 exons except that GaCBL3-2, GrCBL10-1, GrCBL10-2 had 9 and GaCBL9, GrCBL1-2 had 7. The majority of GhCBLs carried 7-11 exons, but GhCBL4-4 had 3 exons and GhCBL3-6 had 22 exons (Fig. 2a).

The putative domains in the Gossypium CBL proteins were also investigated. EF-hand motifs, which bind to $\mathrm{Ca}^{2+}$ ions to transfer calcium signals, were observed in all CBL members. Each CBL proteins had 3 EF-hand motifs except for GaCBL9, which contained 2 such motifs (Fig. 2A). Furthermore, a conserved myristoylation motif (MGCXXS/T) was detected in the Nterminal regions of $11 \mathrm{CBL}$ proteins. These proteins included 4 GaCBLs, 2 GrCBLs and 5 GhCBLs (Fig. 2B, C). A conserved palmitoylation site with N-terminal Cys residue at third, fourth, fifth or sixth position in amino acid sequence also existed in many cotton CBL members. The two sites are important in the attachment of a protein to membrane (Mohanta et al., 2015). 

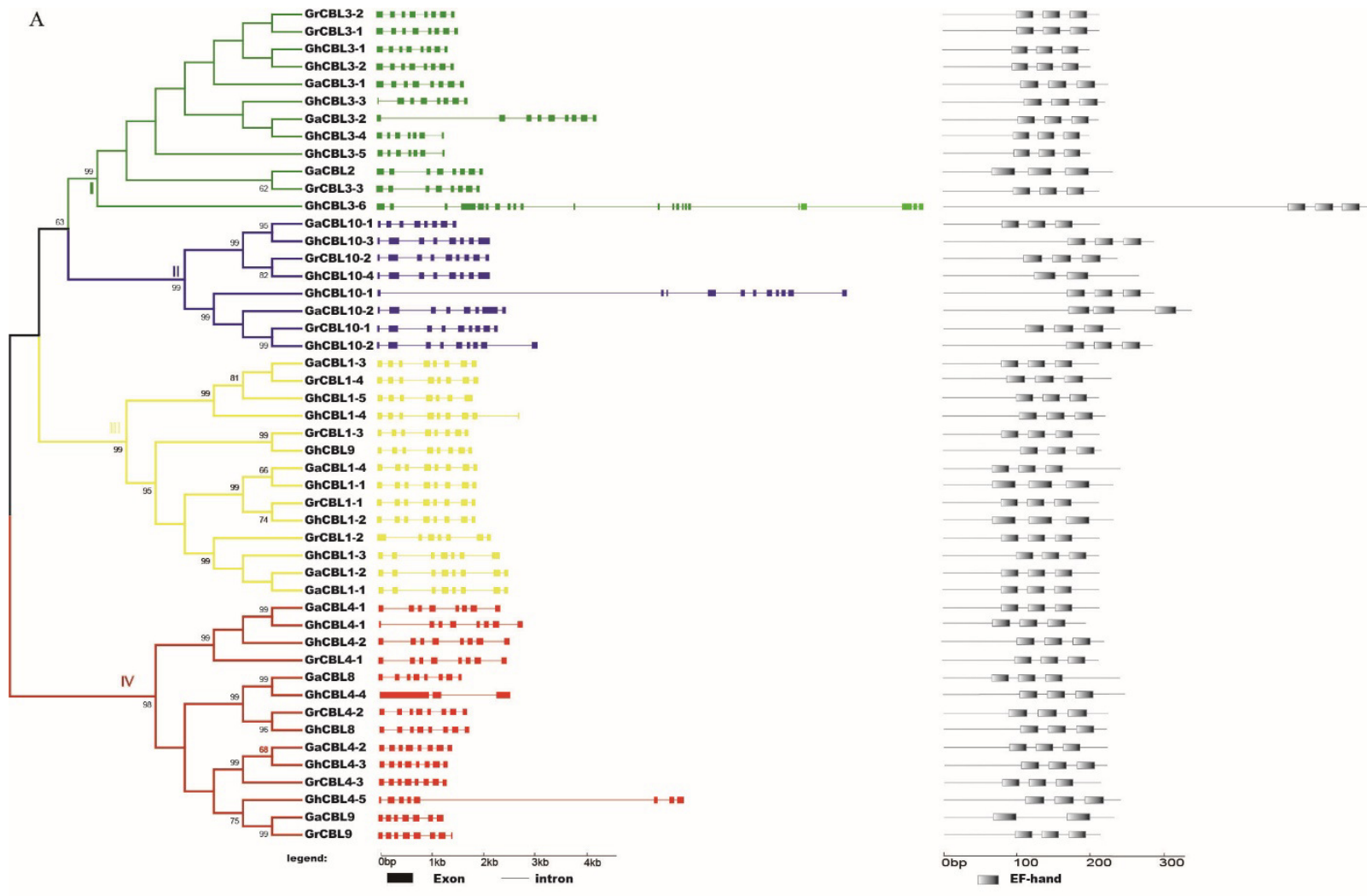

B

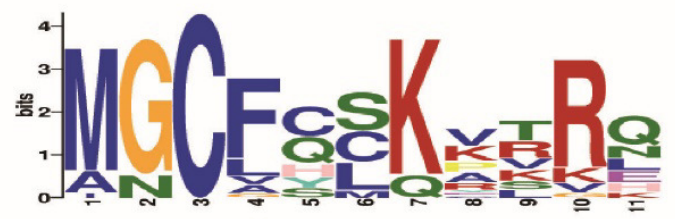

$\mathrm{C}$

\begin{tabular}{|c|c|c|}
\hline Name & Start & Myristoylation site \\
\hline GaCBL1-1 & 1 & MGCFHSKVTRQ HPGYEDPVTL \\
\hline GaCBL1-2 & 1 & MGCF ISKVTRQ HPGYEDPVTL \\
\hline GaCBL1-4 & 1 & MGCFQSKVTRQ YPGHEDPI IL \\
\hline GaCBL4-1 & 1 & MGCASSKRSKQ LHGYRDPSVL \\
\hline GrCBL1-1 & 1 & MGCFQSKVTRQ YPGHEDPI IL \\
\hline GrCBL4-1 & 1 & MGCGSSKRSKQ LHGYEDPSVL| \\
\hline GhCBL1-3 & 1 & MGCF SKVTRQ HPGYEDPVTL \\
\hline GhCBL1-1 & 1 & MCCFQSKVTRQ YPGHEDPIIL \\
\hline GhCBL1-2 & 1 & MGCFQSKVTRQ YPGHEDPI IL \\
\hline GhCBL4-2 & 1 & MGCASSKRSKO LHGYEDPSVL \\
\hline GhCBL9 & 1 & MGCF SKV TRH BDP IE LA SQT \\
\hline
\end{tabular}

Fig. 2 Analysis of phylogenetic relationship, gene architecture and conserved domains of CBLs in Gossypium

(A) The phylogenetic tree, exon-intron architecture and EF-hand domains of CBLs in $G$. arboreum, G. raimondii and G. hirsutum. The four major subfamilies are numbered I to IV. The color boxes indicate exons, and the color lines indicate introns; (B) The logo of the myristoylation motif, the capital letters stand for the amino acids, the higher the letter, the higher 
the conservation; (C) Multiple sequences containing the myristoylation motif in Gossypium CBLs.

\section{Synteny analysis of CBL genes in Gossypium}

241 To investigate the genetic origins and evolution of the CBLs in Gossypium, the homologous gene

242 pairs among the $C B L s$ from $G$. arboretum, G. raimondii and G. hirsutum were monitored, and 243 the collinear analysis was carried out. The results revealed that 10 homologous gene pairs existed 244 between $G$. arboreum and G. hirsutum, and 11 homologous gene pairs were found between $G$. 245 raimondii and G. Hirsutum (Fig. 3A). Using the same method, 7 homologous gene pairs were 246 observed between $G$. arboreum and G. raimondii. They were distributed on 5 chromosomes in $G$. 247 arboreum and 5 chromosomes in G. raimondii, respectively (Fig. 3B). Moreover, 212 248 homologous gene pairs (both based on orthology and paralogy) were found among the CBLs 249 from the 3 Gossypium species (Table S 1). These results imply that many cotton CBL genes may 250 have evolved through segmental duplication.
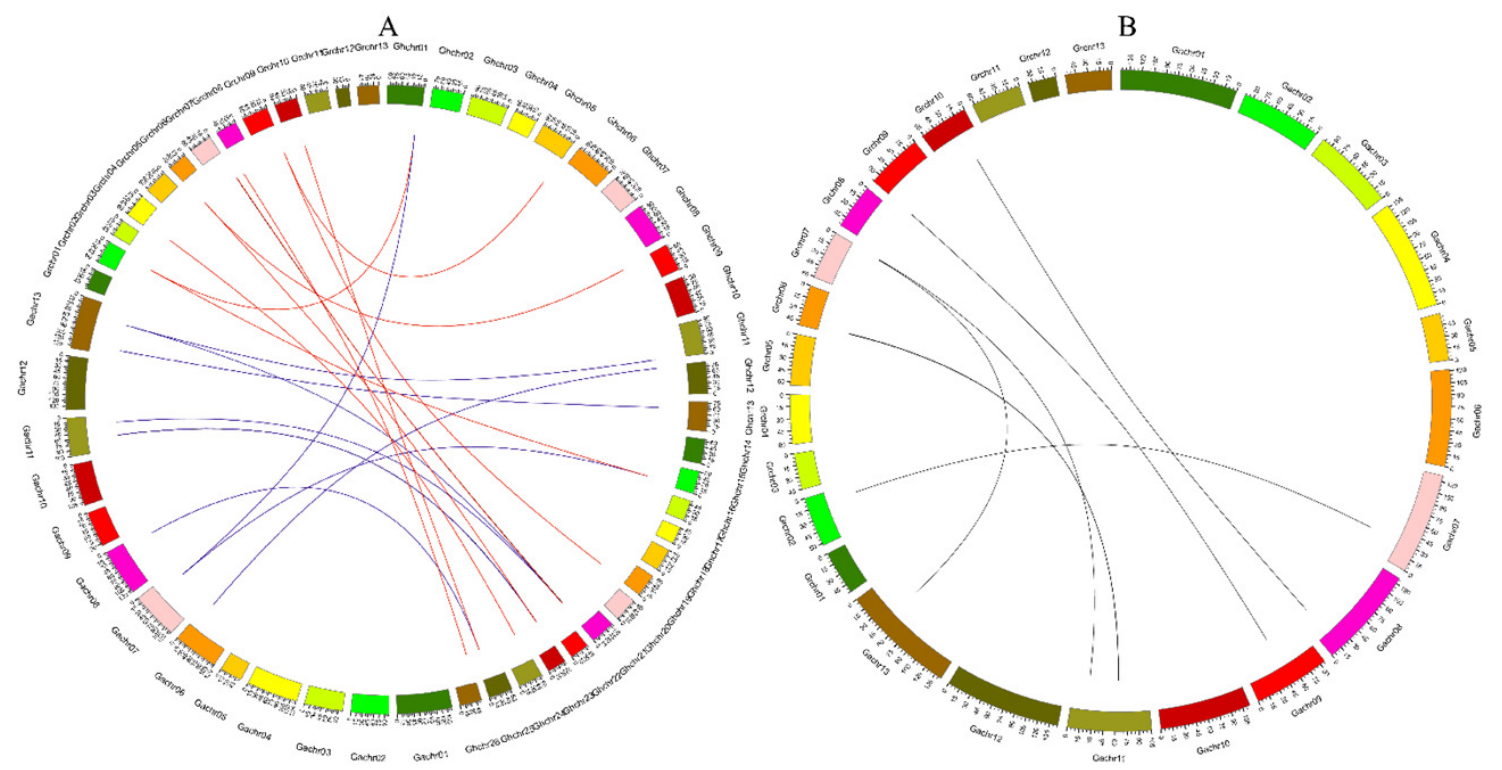

Fig. 3 Genome-wide synteny analysis of Gossypium CBL genes

(A) Synteny analysis between $G$. hirsutum and two diploid species G. arboreum and $G$. raimondii. Blue lines link gene pairs between G. arboreum and G. hirsutum, and red lines link gene pairs between G. raimondii and G. hirsutum; (B) Synteny analysis between G. arboreum and G. raimondi. 


\section{Analysis of $\mathrm{Ka} / \mathrm{Ks}$ values of the CBLs}

252

253

254

255

256

257

To better understand the divergence of the Gossypium CBL genes after polyploidization, the value $\mathrm{Ka}$ and $\mathrm{Ks}$ and their ratio $(\mathrm{Ka} / \mathrm{Ks})$ were evaluated for the homologous gene pairs among $G$. arboreum, G. raimondii and G. hirsutum (Fig. 4,Table S 2). The results showed that the $\mathrm{Ka} / \mathrm{Ks}$ values among most of the homologous genes were less than 1, indicating they evolved under the purifying selection effect. Only GhCBL10-2/GrCBL10-1 has a $\mathrm{Ka} / \mathrm{Ks}$ ratio more than 1 , hinting that the gene pair may have been generated via the directional selection.

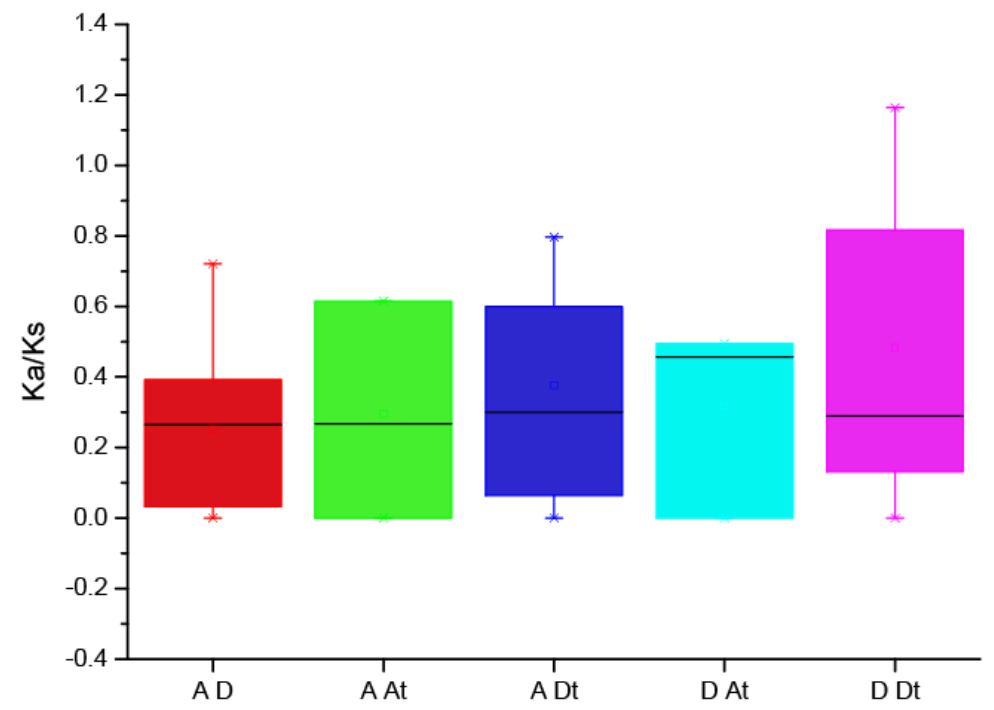

Fig 4. The $\mathrm{Ka} / \mathrm{Ks}$ values of the $\mathrm{CBL}$ homologous genes between the A genome, $\mathrm{D}$ genome and subgenomes of $G$. hirsutum $\left(\mathrm{A}_{\mathrm{t}} \mathrm{D}_{\mathrm{t}}\right)$

\section{Phylogenetic relationship of CBLs in Gossypium and other plant species}

To gain insight into the evolutionary relationships among GaCBLs, GrCBLs, GhCBLs and CBLs of other plant species, we constructed a phylogenetic tree. Full-length amino acid sequences of

260126 predicted CBL proteins were obtained from G. arboretum, G. raimondii, G. hirsutum, $A$.

261 thaliana, C. papaya, G. max, $V$. vinifera, T. cacao, P. trichocarpa, R. communis and O. sativa.

262 Phylogenetic trees were generated using the neighbor-joining method and MEGA 5.0 software. 
263 The CBLs family was divided into thirteen subfamilies according to the topology of the

264 phylogenetic tree (Fig.5). As expected, the three Gossypium CBLs commonly clustered closely

265 in a subfamily. Most of them belonged to subfamily two, eight and thirteen. We found that the

266 CBL members from different dicotyledon species and rice always clustered in a subfamily,

267 suggesting that the CBLs shared an ancestral sequence before the divergence of eudicots and

268 monocots or convergent evolution events for these CBLs might have occurred in adaptations to

269 drastic changes in the environment. Moreover, the CBLs from Gossypium plants often clustered

270 together with those from T. cacao (Fig.5). These results are expected because both Gossypium

271 and T. cacao are in the Malvaceae family.

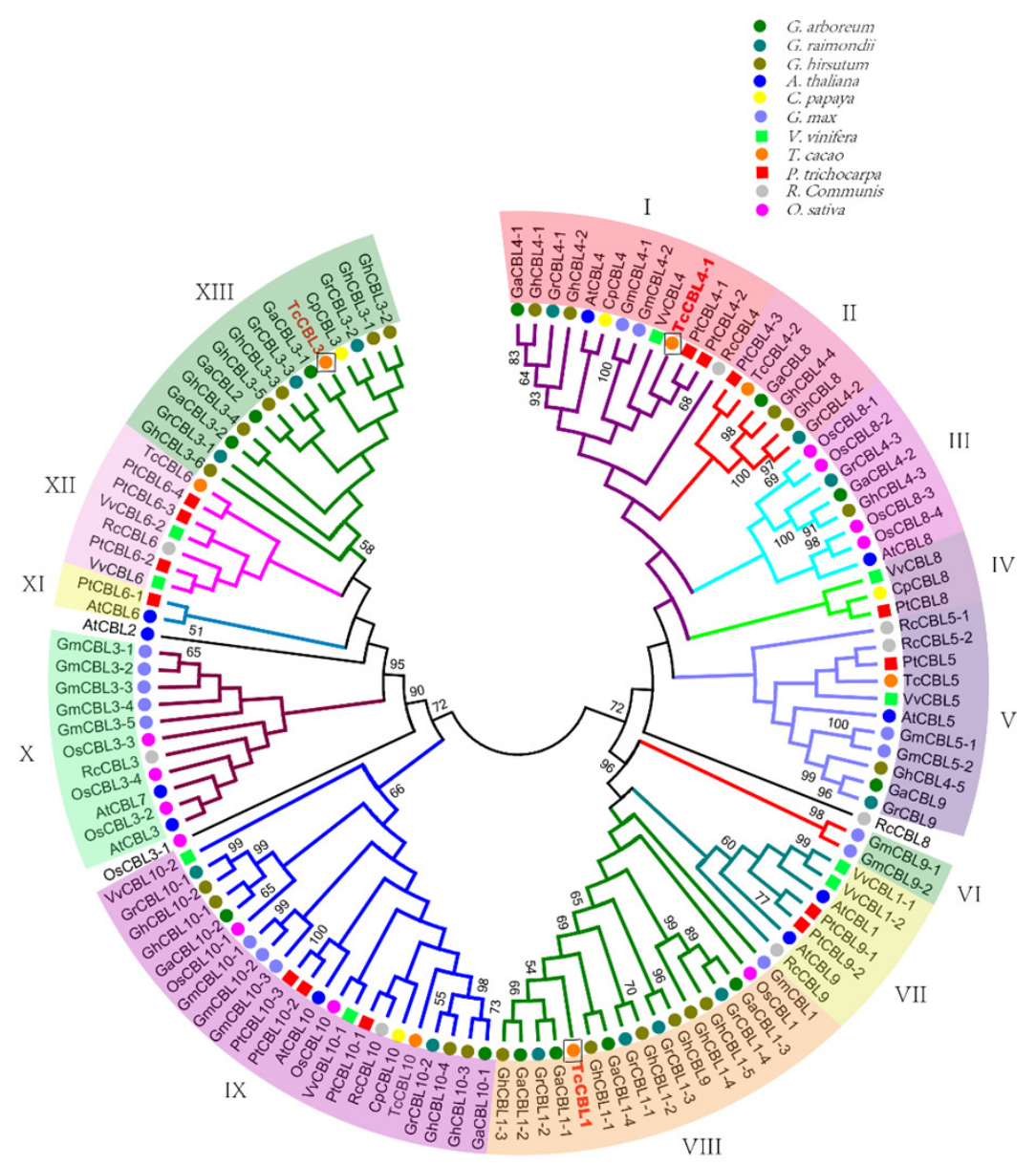

Fig. 5 Phylogenetic tree of CBLs in Gossypium and other plant species

The plants in the square frame indicated that the CBL genes outside of Gossypium have the closest evolutionary relationship with Gossypium CBLs. 
272 Annotation analysis of GhCBLs

273 Putative functions of GhCBLs were analyzed using KOG (EuKaryotic orthologous groups (KOG)

274 database (ftp://ftp.ncbi.nih.gov/pub/COG/KOG). Only the information on GhCBL3-6 was

275 obtained. It was predicted that GhCBL3-6 played roles in modulation of RNA processing and

276 modification, signal transduction, and coenzyme transport and metabolism. Gene ontology (GO)

277 database for the 22 GhCBLs was also assessed. The result showed that these GhCBL members

278 were capable of binding calcium ion, like those of other plant species. These analyses indicate

279 that GhCBLs and other CBLs are of great importance in $\mathrm{Ca}^{2+}$ signal transduction in plants.

280 Expression analysis of $G h C B L$ genes in tissues

281 The expression patterns of all the 22 GhCBL genes in tissues were monitored by qRT-PCR. We

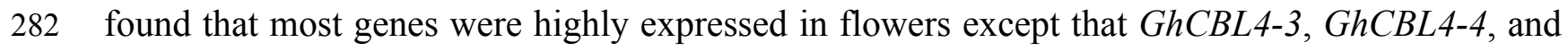
$283 G h C B L 8$ were dominantly expressed in roots and GhCBL3-6 strongly expressed in leaves.

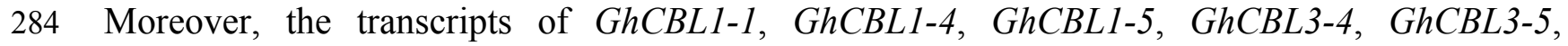
285 GhCBL3-6 and GhCBL9 were relatively abundant in fiber, and those of GhCBL4-3 were also


287 may mainly function in roots, GhCBL3-6 mainly functions in leaves and other genes may chiefly 288 act in flowers. GhCBL1-1, GhCBL1-4, GhCBL1-5, GhCBL3-4, GhCBL3-5, GhCBL3-6 and $289 G h C B L 9$ also probably play a part in fiber development in cotton. 

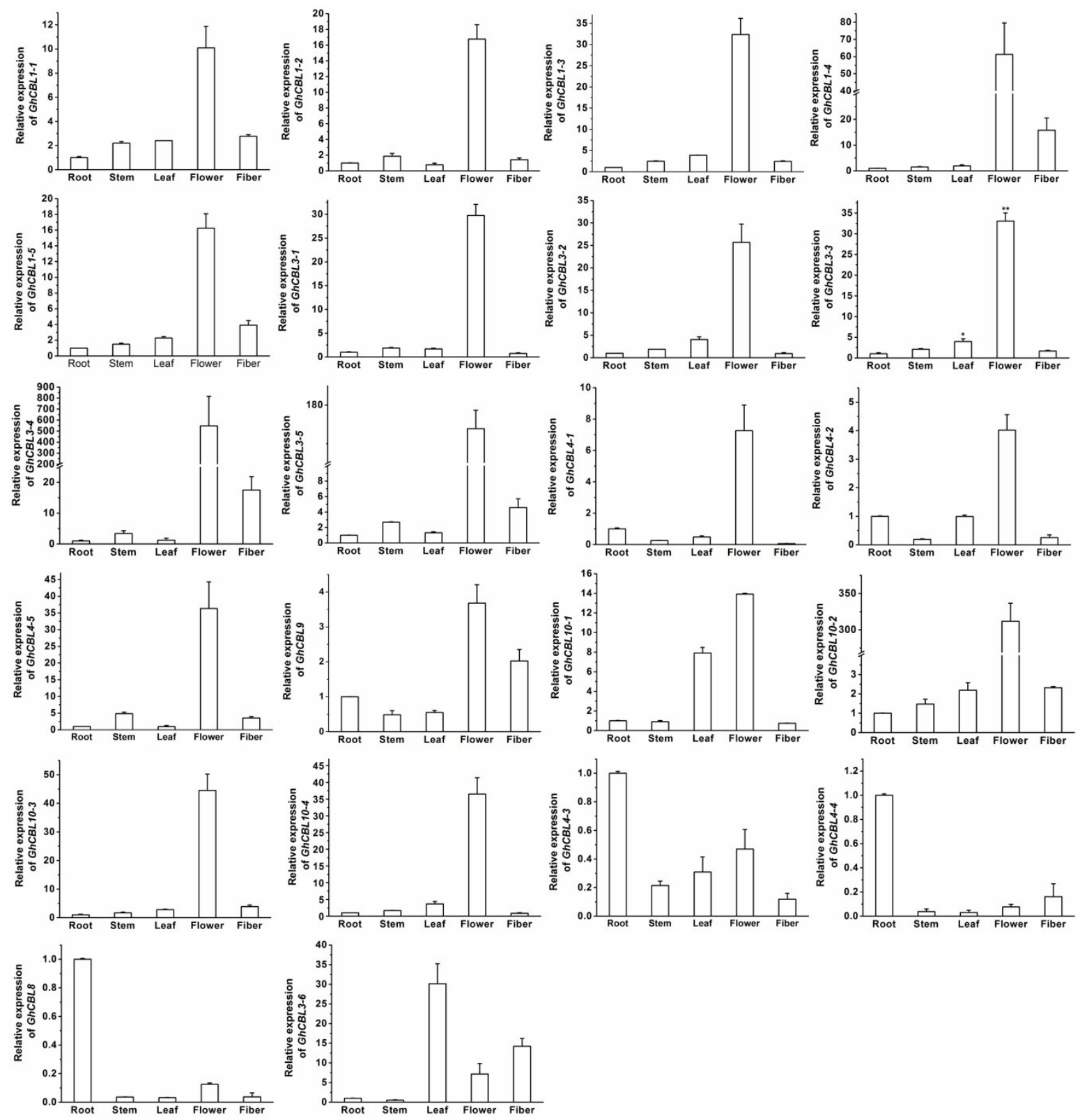

Fig. 6 Expression of 22 GhCBL genes in tissues of cotton

The relative expression of genes was calculated from 3 independent replicates. The expression value of the gene in roots was set as 1 . The vertical bars represent the standard error.

\section{Expression patterns of $G h C B L s$ in responding to potassium deficiency}

291 CBLs have been addressed to play key roles in response to $\mathrm{K}^{+}$deprivation in Arabidopsis and rice (Li et al., 2014a; Mao et al., 2016). Accordingly, we measured the expression patterns of the $22 G h C B L$ genes in response to potassium deficiency. As a whole, potassium deficiency 
294 moderately altered the expression levels of $G h C B L$ genes (Fig. 7). Under potassium deficiency, 295 the transcripts of many genes were reduced at $6 \mathrm{~h}$, but increased at $2 \mathrm{~d}$ and/or $5 \mathrm{~d}$. These gene 296 included GhCBL3-1, GhCBL3-2, GhCBL3-3, GhCBL3-4, GhCBL4-4, and GhCBL10-3. The 297 expression levels of $G h C B L 3-5, G h C B L 3-6, G h C B L 4-3, G h C B L 4-5, G h C B L 8$ and $G h C B L 9$ were 298 decreased while those of other genes were unchanged after shortage of potassium (Fig. 7). The 299 effects of $\mathrm{K}^{+}$resupply on the abundances of $G h C B L$ transcripts were also investigated.

300 Compared with $5 \mathrm{~d}$ of low- $\mathrm{K}^{+}$treatments, $3 \mathrm{~h}$ of $\mathrm{K}^{+}$refeeding clearly resulted in decreases in the 301 expression of many genes such as $G h C B L 1-3, G h C B L 1-5, G h C B L 3-2, G h C B L 3-3, G h C B L 3-4$, $302 G h C B L 10-1$ and $G h C B L 10-3$. However, $\mathrm{K}^{+}$resupply increased the expression of GhCBL4-1. The 303 transcriptional levels of other genes did not significantly alter upon $\mathrm{K}^{+}$resupply (Fig. 7). These 304 results suggest that a number of GhCBLs may play roles in response to potassium starvation in 305 cotton. 

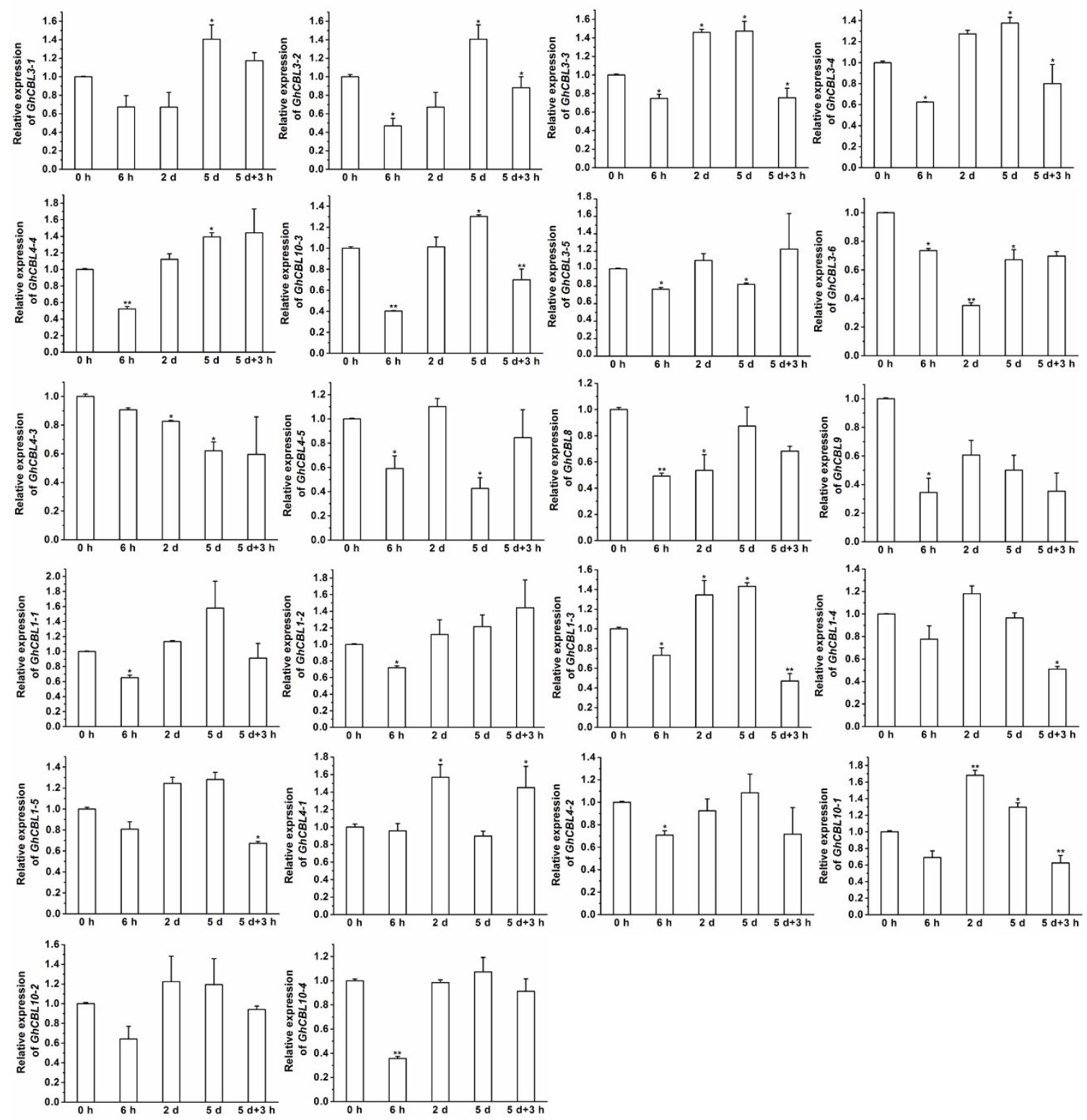

Fig. 7 Expression of 22 GhCBL genes under potassium deprivation

The relative expression of GhCBLs was examined under potassium deficiency or resupply for indicated period of time. The expression value of the gene at $0 \mathrm{~h}$ was set as 1 . The vertical bars mean the standard error. Statistical analyses were conducted by student's $t$ test to assess the differences between the samples at $0 \mathrm{~h}$ and those at $6 \mathrm{~h}, 2 \mathrm{~d}$, or $5 \mathrm{~d}$ as well as between the samples at $5 \mathrm{~d}$ and those upon resupplying potassium for $3 \mathrm{~h}(5 \mathrm{~d}+3 \mathrm{~h})$. The single and double

311 asterisks means that the differences are significant $(P \leqslant 0.05)$ and extremely significant 
$312(P \leqslant 0.01)$, respectively.

\section{Several GhCBLs can interact with GhCIPK23 in vitro}

314 To examine whether GhCBLs interact with GhCIPK23, yeast two-hybrid experiments were 315 performed and total of 12 GhCBLs were measured. Among them, GhCBL1-2, GhCBL1-3, 316 GhCBL4-4, GhCBL8, GhCBL9 and GhCBL10-3 were observed to interact with GhCIPK23.

317 Furthermore, GhCBL1-2 and GhCBL9, the respective homologues of Arabidopsis CBL1 and 318 CBL9, displayed more strong interactive signals with GhCIPK23 in yeast, suggesting that 319 GhCBL1-2 and GhCBL9 may directly regulate GhCIPK23 in cotton.

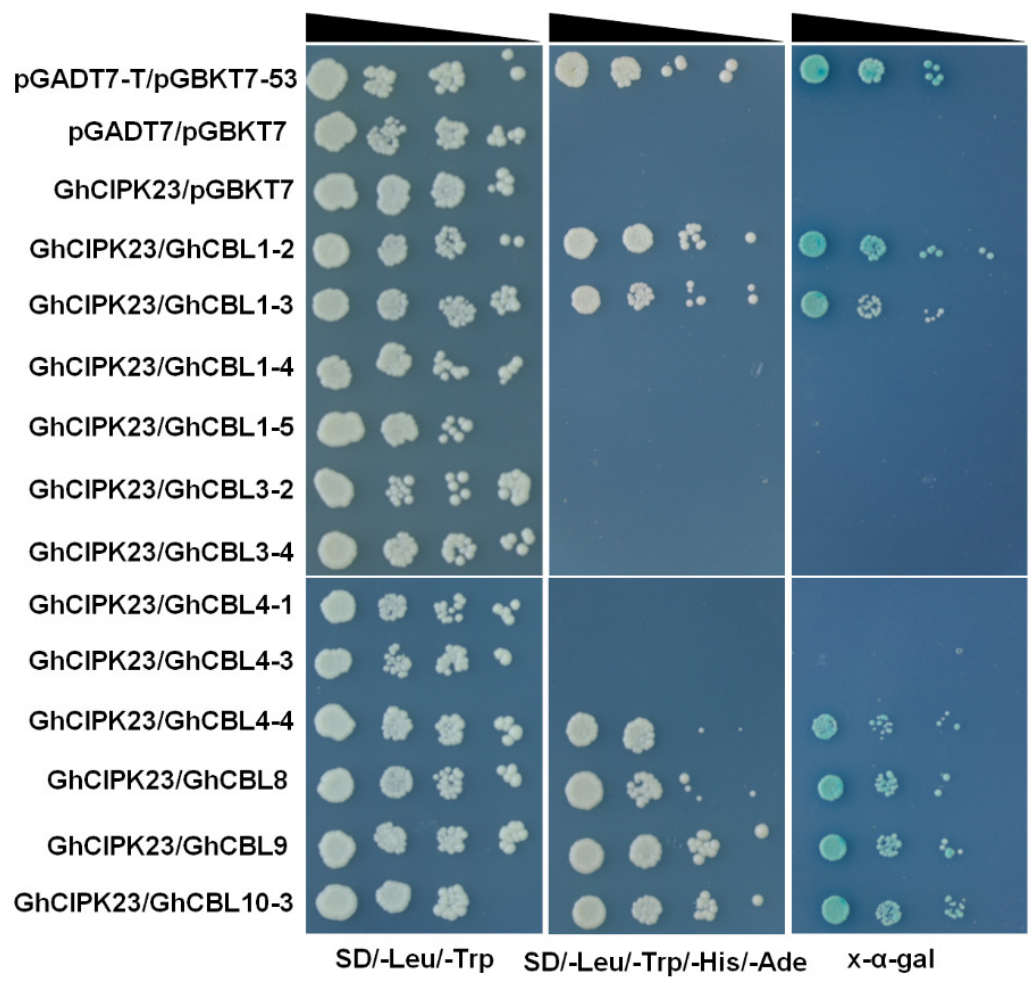

Fig. 8 Yeast two-hybrid analysis of interactions between GhCBLs and GhCIPK23

The yeast cells containing the indicated plasmids were grown on the non-selective SD/-Leu/-Trp solid medium and selective SD/-Leu/-Trp/-His/-Ade solid medium, followed by X- $\alpha$-Gal staining. The reduced cell densities in the dilution series are shown by narrowing triangles when proceeding from left to right. The first row represents a positive control, the $2^{\text {th }}$ and $3^{\text {th }}$ rows represent two negative controls. 
321 In the present study, we identified 13, 13 and 22 CBL genes in G. arboreum, G. raimondii and $G$.

322

323

324

325

326

327

328

329

330

331

332

333

334

335

336

337

338

339

340

341

342

343

344

345

346

347

hirsutum genomes, respectively (Table 3). Among the 22 GhCBL genes, 11 and 11 were assigned to the $A_{t}$ and $D_{t}$ subgenome, respectively. They were similar to the number of $C B L s$ found in G. arboreum and G. raimondii, respectively. We detected that 8 GaCBLs and 9 GrCBLs were homologous genes of GhCBLs. However, homologues of 5 GaCBLs and 4 GrCBLs were not discovered in the genome of G. hirsutum. These findings indicate that the $8 \mathrm{GaCBLs}$ and 9 $G r C B L s$ have been maintained in $G$. hirsutum after polyploidization event, while the 5 GaCBLs and 4 GrCBLs diverged from their orthologs in G. hirsutum during evolution. Moreover, we observed 5 GhCBLs (GhCBL1-3, GhCBL3-5, GhCBL4-1, GhCBL4-4, GhCBL10-1) in $\mathrm{A}_{\mathrm{t}}$ subgenome and 2 GhCBLs (GhCBL3-4, GhCBL3-6) in $\mathrm{D}_{\mathrm{t}}$ had no homologues in A genome of $G$. arboreum and D genome of $G$. raimondii, respectively. It is conceivable because selection pressures in diploids per loci are different than in the allotetraploid. Relaxed selection allows for development of novel and new functional alleles, but may also accumulate non functional, both at a higher rate possible that within the diploids. G. arboreum originates in the Africa/Arabia while G. raimondii and G. hirsutum originate in the Americas (Wendel et al., 2010). They are distributed in quite different places during evolution. Moreover, G. arboreum and G. hirsutum are two domasticated species (Wendel et al., 2010). Hence, geographic separation of the three species, and human selection may be essential for the diversity of the CBLs in Gossypium. The physical properties of most GaCBLs and GrCBLs were similar to those of GhCBLs (Table 3), suggesting that the functions of the CBLs from the three cotton species remained highly conserved during evolution. The majority of Gossypium CBLs was predicted to localize in the membrane, just like many CBLs in Arabidopsis and rice. In Arabidopsis, CBL1 and CBL9 were described to localize in the PM. CBL2, CBL3 and CBL6 localize in tonoplast whereas CBL10 is in both PM and tonoplast (Mao et al., 2016). Rice CBL1 is also present in PM. The localizations of the CBLs should be consistent with their primary roles of sensing and transferring $\mathrm{Ca}^{2+}$ signals in Gossypium. However, GhCBL3-6 was predicted to be nuclear. Its roles are unknown at present. Experimental characterization of GhCBL3-6 might shed light on 
348

349

350

351

352

353

354

355

356

357

358

359

360

361

362

363

364

365

366

367

368

369

370

371

372

373

374

some novel functions of it. GhCBL3-6 also gives obvious proof of the evolutionary advantage of being tetraploid. It may be a product of significant human intervention because nothing like it was seen in either diploid.

Analysis of gene distributions on chromosomes showed that most homologues of GaCBLs and GrCBLs in G. hirsutum were present in their corresponding $\mathrm{A}_{\mathrm{t}}$ and $\mathrm{D}_{\mathrm{t}}$ homologous chromosomes, respectively. These findings indicate that $G h C B L s$ originate from DNA polyploidization.

However, some GhCBLs homologues of GaCBLs and GrCBLs did not appear on their corresponding $A_{t}$ or $D_{t}$ chromosomes, suggesting that complex exchange events of chromosome segments occurred in G. hirsutum during evolution. Additionally, separated (e.g. GaCBL4-1 and GaCBL4-2; GrCBL1-1 and $G r C B L 1-2)$ and jointed (GaCBL4-2 and $G a C B L 9)$ distributions of the Gossypium CBL homologous genes in chromosomes in combination with the colinearity results of these genes (Fig. 1; Fig. 3) imply that both segmental duplication and tandem duplication are essential for the generation of cotton CBLs during genetic evolution. The number of introns in coding region of most CBL genes in Gossypium was six or seven, very similar to that in CBLs genes in Arabidopsis, rice, maize, wheat, canola and eggplant (Kolukisaoglu et al., 2004; zhang et al., 2014; Sun et al., 2015; Li et al., 2016; Zhang et al., 2016), reflecting the rather conserved structure of CBL genes in different species. Moreover, nearly all of the Gossypium CBLs shared three conserved EF hand domains with other higher plants (Fig. 2). In addition, many $C B L s$ from Gossypium contained the myristoylated and palmitoylated sites, which may facilitate the targeting of CBL-CIPK complex to membrane. These features are also similar to those in Arabidopsis, rice and other plants (Kolukisaoglu et al., 2004; Mohanta et al., 2015). The conserved structure of these CBL family members in different plants might reflect a very similar mode of action and/or conserved interaction with their target protein CIPKs (Mohanta et al., 2015).

Measurement of the ratio of Ka to Ks indicated that majority of Gossypium CBL homologous genes have undergone purifying selection whereas GhCBL10-2/GrCBL10-1 has experienced directional selection after polyploidization (Fig. 4). These results suggest that most GhCBLs have 
375

376

377

378

379

380

381

382

383

384

very high similarity in gene sequences and highly conserved functions to their orthologs from $G$. arboretum and G. raimondii during evolution. By contrast, a large divergence between GhCBL10-2 and GrCBL10-1G has happened. GhCBL10-2 may have evolved some novel functions through natural selection and human selection.

Phylogenetic analysis results revealed that the CBLs in Gossypium have closer relationship with those in cocoa than in other plants tested (Fig. 5). These findings strongly suggest that the cotton species may have a more recent common ancestor with cacao relative to other plant species, in line with the results of other gene families in Gossypium (Li et al., 2014b; Li et al., 2016). It may justify using CBL as another evolutionary model in plants because it showed highest similarity with another taxon from the same family and may help to narrow down the most vital or evolutionarily conserved or ancient sequences in Gossypium.

Expression analysis results showed that almost all of the $G h C B L$ genes were expressed in various tissues including the root, stem, leaf, flower and fiber. Of note, most genes were dominantly expressed in the flower and fiber (Fig. 6), hinting that these genes may play important roles in the reproductive development in cotton. G. hirsutum is a highly domesticated plant for its seed fiber, which is developed from the flower. Preferential expression of many GhCBLs in flowers and fibers suggests that human selection markedly affects the genetic variation and expression profiles of GhCBLs. Besides, the expression levels of GhCBL4-3 GhCBL4-4 and GhCBL8 in roots were clearly higher than those of other genes. These data imply that the three genes may function in modulation of ion transport or acclimation to diverse abiotic stresses in roots. Their detailed actions and mechanisms will be examined in the future.

The expression of 22 GhCBLs in responding to potassium starvation was determined. The transcription of most genes was moderately promoted at $2 \mathrm{~d}$ and/or $5 \mathrm{~d}$ post low-potassium treatments (Fig. 7), indicating multiple GhCBL genes likely regulate cotton response to potassium deprivation. Strikingly, in Arabidopsis, the expression of CBL1 and CBL9 was reported to be stable, and the transcripts of $C B L 10$ in roots were moderately decreased under low-potassium conditions (Cheong et al., 2007; Ren et al., 2013). These results imply that 
402

403

404

405

406

407

408

409

410

411

412

413

414

415

416

417

418

419

420

421

422

423

424

425

426

427

428

constitutive expression of some $C B L$ genes may be enough for transmitting $\mathrm{Ca}^{2+}$ signals to downstream targets in response to potassium deficiency in plants. Thus, those $G h C B L s$ that were not induced by low-potassium stress also likely play a part in adaptations to potassium deprivation in cotton. However, which sequences and how GhCBLs regulate potassium starved responses remains to be investigated in the future.

CIPK23 has been observed to function in diverse cellular processes in Arabidopsis (Mao et al., 2016). In this study, 6 out of 12 GhCBLs could interact with GhCIPK23 in yeast (Fig. 8), indicating that different GhCBL members may interact with and modulate GhCIPK23 in various growth and/or stress responses in cotton. The cotton homologues of Arabidopsis CBL1 and CBL9 suggest that GhCBL1 and GhCBL9 probably play similar roles to CBL1 and CBL9 in cotton.

\section{ACKNOWLEDGEMENTS}

This work was supported by the Science and Technology Development Program of He'nan in China (162102110005) and Foundation of He'nan Educational Committee of China (15A210018, 17A180018 and 14B180029).

\section{REFERENCES}

Allen RD. 2010. Opportunities for engineering abiotic stress tolerance in cotton plants. Cotton. Berlin:Springer Verlag, 127-160.

Cheong YH, Pandey GK, Grant JJ, Batistic O, Li L, Kim BG, Lee SC, Kudla J, Luan S. 2007. Two calcineurin B-like calcium sensors, interacting with protein kinase CIPK23, regulate leaf transpiration and root potassium uptake in Arabidopsis. The Plant Journal 52:223-239 DOI 10.1111/j.1365-313X.2007.03236.x.

Cho JH, Lee JH, Park YK, Choi MN, Kim KN. 2016. Calcineurin B-like protein CBL10 directly interacts with TOC34 (Translocon of the Outer membrane of the Chloroplasts) and decreases its GTPase activity in Arabidopsis. Frontiers in Plant Science 7:1911 DOI 10.3389/fpls.2016.01911.

Drerup MM, Schlücking K, Hashimoto K, Manishankar P, Steinhorst L, Kuchitsu K, 
Kudla J. 2013. The calcineurin B-like calcium sensors CBL1 and CBL9 together with their interacting protein kinase CIPK26 regulate the Arabidopsis NADPH oxidase RBOHF. Molecular Plant 6:559-569 DOI 10.1093/mp/sst009.

Finn RD, Mistry J, Tate J, Coggill P, Heger A, Pollington JE, Gavin OL, Gunasekaran P, Ceric G, Forslund K, Holm L, Sonnhammer EL, Eddy SR, Bateman A. 2010. The pfam protein families database. Nucleic Acids Research 38:D211-22 DOI 10.1093/nar/gkp985.

Fuglsang AT, Guo Y, Cuin TA, Qiu Q, Song C, Kristiansen KA, Bych K, Schulz A, Shabala S, Schumaker KS, Palmgren MG, Zhu JK. 2007. Arabidopsis protein kinase PKS5 inhibits the plasma membrane $\mathrm{H}^{+}$-ATPase by preventing interaction with 14-3-3 protein. Plant Cell 19:1617-1634 DOI 10.1105/tpc.105.035626.

Gao P, Zhao PM, Wang J, Wang HY, Du XM, Wang GL, Xia GX. 2008. Co-expression and preferential interaction between two calcineurin B-like proteins and a CBL-interacting protein kinase from cotton. Plant Physiology and Biochemistry 46:935-940 DOI 10.1016/j.plaphy.2008.05.001.

Held K, Pascaud F, Eckert C, Gajdanowicz P, Hashimoto K, Corratgé-Faillie C, Offenborn JN, Lacombe B, Dreyer I, Thibaud JB. 2011. Calcium-dependent modulation and plasma membrane targeting of the AKT2 potassium channel by the CBL4/CIPK6 calcium sensor/protein kinase complex. Cell Research 21:1116-1130 DOI 10.1038/cr.2011.50.

Ho CH, Lin SH, Hu HC, Tsay YF. 2009. CHL1 functions as a nitrate sensor in plants. Cell 138:1184-1194 DOI 10.1016/j.cell.2009.07.004.

Kim BG, Waadt R, Cheong YH, Pandey GK, Dominguez-Solis JR, Schültke S, Lee SC, Kudla J, Luan S. 2007. The calcium sensor CBL10 mediates salt tolerance by regulating ion homeostasis in Arabidopsis. Plant Journal 52:473-484 DOI 10.1111/j.1365313X.2007.03249.x.

Kolukisaoglu Ü, Weinl S, Blazevic D, Batistic O, Kudla J. 2004. Calcium sensors and their interacting protein kinases: Genomics of the Arabidopsis and Rice CBL-CIPK signaling networks. Plant Physiology 134:43-58 DOI 10.1104/pp.103.033068. 
456

457

458

459

460

461

462

463

464

465

466

467

468

469

470

471

472

473

474

475

476

477

478

479

480

481

482

Kudla J, Batistič O, Hashimoto K. 2010. Calcium signals: the lead currency of plant information processing. The Plant Cell 22:541-563 DOI 10.1105/tpc.109.072686.

Kudla J, Xu Q, Harter K, Gruissem W, Luan S. 1999. Genes for calcineurin B-like proteins in Arabidopsis are differentially regulated by stress signals. Proceedings of the National Acadamy of Sciences of the United States of America 96:4718-4723.

Larkin MA, Blackshields G, Brown NP, Chenna R, McGettigan PA, McWilliam H, Valentin F, Wallace IM, Wilm A, Lopez R, Thompson JD, Gibson TJ, Higgins DG. 2007. Clustal $\mathrm{W}$ and Clustal $\mathrm{X}$ version 2.0. Bioinformatics 23:2947-2948 DOI 10.1093/bioinformatics/btm404.

Li F, Fan G, Wang K, Sun F, Yuan Y, Song G, Li Q, Ma Z, Lu C, Zou C, Chen W, Liang X, Shang H, Liu W, Shi C, Xiao G, Gou C, Ye W, Xu X, Zhang X, Wei H, Li Z, Zhang G, Wang J, Liu K, Kohel RJ, Percy RG, Yu JZ, Zhu YX, Wang J, Yu S. 2014b. Genome sequence of the cultivated cotton Gossypium arboreum. Nature Genetics 46:567-572 DOI 10.1038/ng.2987.

Li J, Jiang MM, Ren L, Liu Y, Chen HY. 2016. Identification and characterization of CBL and CIPK gene families in eggplant (Solanum melongena L.). Molecular Genetics and Genomics 291:1769-1781 DOI 10.1007/s00438-016-1218-8.

Li J, Long Y, Qi GN, Li J, Xu ZJ, Wu WH, Wang Y. 2014a. The Os-AKT1 channel is critical for $\mathrm{K}^{+}$uptake in rice roots and is modulated by the rice CBL1-CIPK23 complex. The Plant Cell 26:3387-3402 DOI 10.1105/tpc.114.123455.

Li L, Kim BG, Cheong YH, Pandey GK, Luan S. 2006. A $\mathrm{Ca}^{2+}$ signaling pathway regulates a $\mathrm{K}^{+}$channel for low-K response in Arabidopsis. Proceedings of the National Acadamy of Sciences of the United States of America 103:12625-12630 DOI 10.1073/pnas.0605129103.

Li W, Shang H, Ge Q, Zou C, Cai J, Wang D, Fan S, Zhang Z, Deng X, Tan Y, Song W, Li P, Jamshed M, Lu Q, Gong W, Li J, Shi Y, Chen T, Gong J, Liu A, Yuan Y. 2016. Genome-wide identification, phylogeny, and expression analysis of pectin methylesterases reveal their major role in cotton fiber development. BMC Genomics 17:1000 DOI 
483

484

485

486

487

488

489

490

491

492

493

494

495

496

497

498

499

500

501

502

503

504

505

506

507

508

509

10.1186/s12864-016-3365-z.

Ligaba-Osena A, Fei Z, Liu J, Xu Y, Shaff J, Lee SC, Luan S, Kudla J, Kochian L, Piñeros M. 2017. Loss-of-function mutation of the calcium sensor CBL1 increases aluminum sensitivity in Arabidopsis. New Phytologist 214:830-841 DOI 10.1111/nph.14420.

Liu LL, Ren HM, Chen LQ, Wang Y, Wu WH. 2013. A protein kinase, calcineurin B-like protein-interacting protein kinase9, interacts with calcium sensor calcineurin B-like protein3 and regulates potassium homeostasis under low-potassium stress in Arabidopsis. Plant Physiology 161:266-277 DOI 10.1104/pp.112.206896.

Luan S. 2009. The CBL-CIPK network in plant calcium signaling. Trends in Plant Science 14:37-42 DOI 10.1016/j.tplants.2008.10.005.

Mao J, Manik SMN, Shi S, Chao J, Jin Y, Wang Q, Liu H. 2016. Mechanisms

and physiological roles of the CBL-CIPK networking system in Arabidopsis thaliana. Genes 7:62 DOI 10.3390/genes7090062.

Mohanta TK, Mohanta N, Mohanta YK, Parida P, Bae H. 2015. Genome-wide identification of calcineurin B-Like (CBL) gene family of plants reveals novel conserved motifs and evolutionary aspects in calcium signaling events. Plant Biology 15:189 DOI 10.1186/s12870-015-0543-0.

Murashige T, Skoog F. 1962. A revised medium for rapid growth and bio-assays with tobacco tissue culture. Physiologia Plantarum 15: 473 - 497

Oosterhuis DM, Loka DA, Raper TB. 2013. Potassium and stress alleviation: Physiological functions and management of cotton. Journal of Plant Nutrition and Soil Science 176:331343 DOI 10.1002/jpln.201200414.

Pandey GK, Cheong YH, Kim KN, Grant JJ, Li L, Hung W, D’Angelo C, Weinl S, Kudla J, Luan S. 2004. The calcium sensor calcineurin B-like 9 modulates abscisic acid sensitivity and biosynthesis in Arabidopsis. The Plant Cell 16:1912-1924 DOI 10.1105/tpc.021311.

Ren XL, Qi GN, Feng HQ, Zhao S, Zhao SS, Wang Y, Wu WH. 2013. Calcineurin B-like protein CBL10 directly interacts with AKT1 and modulates $\mathrm{K}^{+}$homeostasis in Arabidopsis. 
The Plant Journal 74:258-266 DOI 10.1111/tpj.12123.

511 Sanyal SK, Pandey A, Pandey GK. 2015. The CBL-CIPK signaling module in plants: a

\begin{abstract}
mechanistic perspective. Physiologia Plantarum 155:89-108 DOI 10.1111/ppl.12344.
\end{abstract}
Sarwat M, Ahmad P, Nabi G, Hu XY. 2013. $\mathrm{Ca}^{2+}$ signals: the versatile decoders of environmental cues. Critical Reviews in Biotechnology 33:97-109 DOI 10.3109/07388551.2012.672398.

Steinhorst L, Mähs A, Ischebeck T, Zhang C, Zhang X, Arendt S, Schültke S, Heilmann I, Kudla J. 2015. Vacuolar CBL-CIPK12 $\mathrm{Ca}^{2+}$-sensor-kinase complexes are required for polarized pollen tube growth. Current Biology 25:1475-1482 DOI 10.1016/j.cub.2015.03.053.

Straub T, Ludewig U, Neuhäuser B. 2017. The kinase CIPK23 inhibits ammonium transport in Arabidopsis thaliana. The Plant Cell 29:409-422 DOI 10.1105/tpc.16.00806.

Sun T, Wang Y, Wang M, Li T, Zhou Y, Wang X, Wei S, He G, Yang G. 2015. Identification and comprehensive analyses of the CBL, and CIPK, gene families in wheat (Triticum aestivum L). Plant Biology 15:269 DOI 10.1186/s12870-015-0657-4.

Tang RJ, Zhao FG, Garcia VJ, Kleist TJ, Yang L, Zhang HX, Luan S. 2015. Tonoplast CBL-CIPK calcium signaling network regulates magnesium homeostasis in Arabidopsis. Proceedings of the National Acadamy of Sciences of the United States of America 112:3134-3139 DOI 10.1073/pnas.142094412.

Thoday-Kennedy EL, Jacobs AK, Roy SJ. 2015. The role of the CBL-CIPK calcium signaling network in regulating ion transport in response to abiotic stress. Plant Growth Regulation 76:3-12 DOI 10.1007/s10725-015-0034-1.

Tian QY, Zhang XX, Yang A, Wang TZ, Zhang WH. 2016. CIPK23 is involved in iron acquisition of Arabidopsis by affecting ferric chelate reductase activity. Plant Science 246:70-79 DOI 10.1016/j.plantsci.2016.01.010.

Wendel JF, Brubaker CL, Seelanan T. 2010. The origin and evolution of Gossypium. Pp 1-18 in: Stewart JM, Oosterhuis D, Heitholt JJ, Mauney JR (Eds.), Physiology of Cotton, 
Berlin: Springer, Netherlands.

538 Xu J, Li HD, Chen LQ, Wang Y, Liu LL, He L, Wu WH. 2006. A protein kinase, interacting 539 with two calcineurin B-like proteins, regulates $\mathrm{K}^{+}$transporter AKT1 in Arabidopsis. Cell $540 \quad$ 125: $1347-1360$.

541 Zhang F, Li L, Jiao Z, Chen Y, Liu H, Chen X, Fu J, Wang G, Zheng J. 2016. 542 Characterization of the calcineurin B-Like (CBL) gene family in maize and functional 543 analysis of ZmCBL9 under abscisic acid and abiotic stress treatments. Plant Science $544 \quad$ 253:118-129 DOI 10.1016/j.plantsci.2016.09.011.

545 Zhang H, Yang B, Liu W-Z, Li H, Wang L, Wang B, Deng M, Liang W, Deyholos MK, 546 Jiang YQ. 2014. Identification and characterization of CBL and CIPK gene families in 547 canola (Brassica napus L.). BMC Plant Biology 14:1 DOI 10.1186/1471-2229-14-8. 